\title{
CRYOGENICS SAFETY REVIEW OF THE ATLAS EXPERIMENT AT CERN
}

\author{
F. Haug, on behalf of the ATLAS collaboration
}

The ATLAS detector at CERN to be installed at $90 \mathrm{~m}$ depth in a 50,000 $\mathrm{m}^{3}$ underground cavern is of unprecedented size and complexity. This is reflected in the helium and nitrogen cryogenic systems required respectively by the magnets (three large superconducting toroids and the central solenoid with 1.6 GJ stored energy) and by the argon calorimeters containing $82 \mathrm{~m}^{3}$ of liquid which can be drained into two $50 \mathrm{~m}^{3}$ dewars in case of emergency. Further coolants of $11 \mathrm{~m}^{3}$ of liquid helium and $15 \mathrm{~m}^{3}$ of liquid nitrogen are stored underground. The potential hazards of the large quantities of cryogens in underground areas require specific attention. Design, construction and quality assurance strictly follow applicable safety rules and the cryogenic process and controls are conceived to actively cope with a number of faults. In severe cases of accidental coolant loss (helium, nitrogen) or argon, detection systems produce alarms which result in the activation of emergency gas extraction. Reviews with international experts confirmed the good safety standard of the systems.

CERN, Accelerator Technology Department, Geneva, Switzerland 


\section{Cryogenics Safety Review of the ATLAS Experiment at CERN}

Haug F., on behalf of the ATLAS collaboration

Accelerator Technology Division, CERN, CH-1211 Geneva 23, Switzerland

The ATLAS detector at CERN to be installed at $90 \mathrm{~m}$ depth in a 50,000 $\mathrm{m}^{3}$ underground cavern is of unprecedented size and complexity. This is reflected in the helium and nitrogen cryogenic systems required respectively by the magnets (three large superconducting toroids and the central solenoid with 1.6 GJ stored energy) and by the argon calorimeters containing $82 \mathrm{~m}^{3}$ of liquid which can be drained into two $50 \mathrm{~m}^{3}$ dewars in case of emergency. Further coolants of $11 \mathrm{~m}^{3}$ of liquid helium and $15 \mathrm{~m}^{3}$ of liquid nitrogen are stored underground. The potential hazards of the large quantities of cryogens in underground areas require specific attention. Design, construction and quality assurance strictly follow applicable safety rules and the cryogenic process and controls are conceived to actively cope with a number of faults. In severe cases of accidental coolant loss (helium, nitrogen) or argon, detection systems produce alarms which result in the activation of emergency gas extraction. Reviews with international experts confirmed the good safety standard of the systems.

\section{INTRODUCTION}

At CERN the $27 \mathrm{~km}$ circumference Large Hadron Collider (LHC) under construction uses large scale cryogenic systems for collider magnets and for two of the detectors (CMS, ATLAS). Basic cryogenic safety issues are addressed in CERN guidelines (1) or in comprehensive handbooks like (2). However, they are not exhaustive for this new endeavour with very large quantities of cryogens used in underground areas. Specific studies were needed and made over the past years at CERN and collaborating institutes to evaluate potential hazards. This permitted the implementation of appropriate safety measures. In particular ATLAS uses large amounts of three different cryogens stored in the proximity of personnel work. This paper summarises the preventive measures to reduce the risk of accidents for the ATLAS experiment.

\section{RISKS}

The largest risks to be found are in the underground areas, in particular in the almost hermetic experimental cavern. A quantity of $82 \mathrm{~m}^{3}$ of liquid argon is permanently stored in the three calorimeter cryostats. A $15 \mathrm{~m}^{3} \mathrm{LN}_{2}$ phase separator dewar is needed for its uninterrupted year-round cooling. The magnet system (toroids and solenoid) operate during approximately 9 months per year with the $11 \mathrm{~m}^{3}$ helium storage dewar at full. In comparison the fluid quantities in the remaining equipment and cooling circuits are small (Table 1).

The three cryogens argon, helium, nitrogen are non toxic fluids. Instead their risks to humans results from their physical properties. At cold even a brief contact with the cryogen can cause severe injuries. The large expansion ratio of the fluids is a potential risk for asphyxiation in confined areas when air oxygen is replaced. An oxygen deficiency hazard (ODH) exists already at oxygen contents of few percent less than the normal $21 \%$ in air. Owing to the different densities at ambient temperatures helium rises, argon accumulates at ground level and nitrogen mixes with air. A liquid spill leads to a mixture of the cryogen with air resulting into the formation of a cold mist with condensed air moisture expanding rapidly with a movement depending on the fluid. Liquid nitrogen and argon fall to ground while a helium air 
mixture moves rapidly upwards. Experiments simulating large liquid helium leak (3) demonstrated the rapid expansion and upward move with mist formation while the tests with argon confirmed the potential danger in particular at the detector floor level (4). The tests revealed that decreasing temperatures and oxygen contents were of concern but that the major problem arises from the cloud formation with impaired visibility for persons trying to use escape routes. The primary objective is, hence, at utmost to avoid cryogen spills by design and process with inherent safety features. However, in the unlikely event of an accident external systems are installed to reduce risks for personnel.
Table 1

\begin{tabular}{|c|c|}
\hline Experimental cavern & quantity \\
\hline Barrel Toroid (BT) & $0.6 \mathrm{~m}^{3} \mathrm{LHe}$ \\
\hline 2 End Cap Toroids (ECT's) & $0.4 \mathrm{~m}^{3} \mathrm{LHe}$ \\
\hline Storage dewar & $11 \mathrm{~m}^{3} \quad \mathrm{LHe}$ \\
\hline Phase seperator cryostat & $1 \mathrm{~m}^{3} \quad \mathrm{LHe}$ \\
\hline Central Solenoid (CS) & $0.02 \mathrm{~m}^{3} \mathrm{LHe}$ \\
\hline Control dewar CS & $0.25 \mathrm{~m}^{3} \mathrm{LHe}$ \\
\hline Barrel Calorimeter (Barrel) & $44 \mathrm{~m}^{3}$ \\
\hline 2 End Cap Calorimeter (EC's) & $38 \mathrm{~m}^{3}$ \\
\hline $2 \times 50 \mathrm{~m}^{3}$ Argon dewars & normally empty \\
\hline $\mathrm{LN}_{2}$ phase separator dewar & $15 \mathrm{~m}^{3} \quad \mathrm{LN}_{2}$ \\
\hline
\end{tabular}

\section{CRYOGENIC SYSTEMS WITH BUILT-IN SAFETY FEATURES}

\section{Cryogenics for magnets}

For the magnets two helium refrigerators are used; the main (MR) and the shield refrigerator (SR) (5). The MR keeps the magnets 600 ton cold mass at $4.5 \mathrm{~K}$. The SR safety function is used in case the MR fails in recovering the vaporised helium from the magnets by one of the two redundant SR compressors. For autonomy of 2 hours during slow discharge the 110001 dewar supplies the toroids via the phase separator cryostat from which the liquid is withdrawn and circulated with one of two redundant pumps. During the 20 minute slow discharge the solenoid is cooled in gravity-driven thermo-syphon mode by 250 1 stored in the control dewar on top of the detector. Figure 1 illustrates this emergency operation with the circuitry of the two refrigerators and the two proximity cryogenic systems. No helium is discharged in the underground cavern. In case of an unlikely accidental magnet energy fast dump the two-phase mixture in the cooling circuits would be expelled without discharging in the underground area (6) to the phase separator cryostat that rises in pressure. All the circuits are designed and tested accordingly.
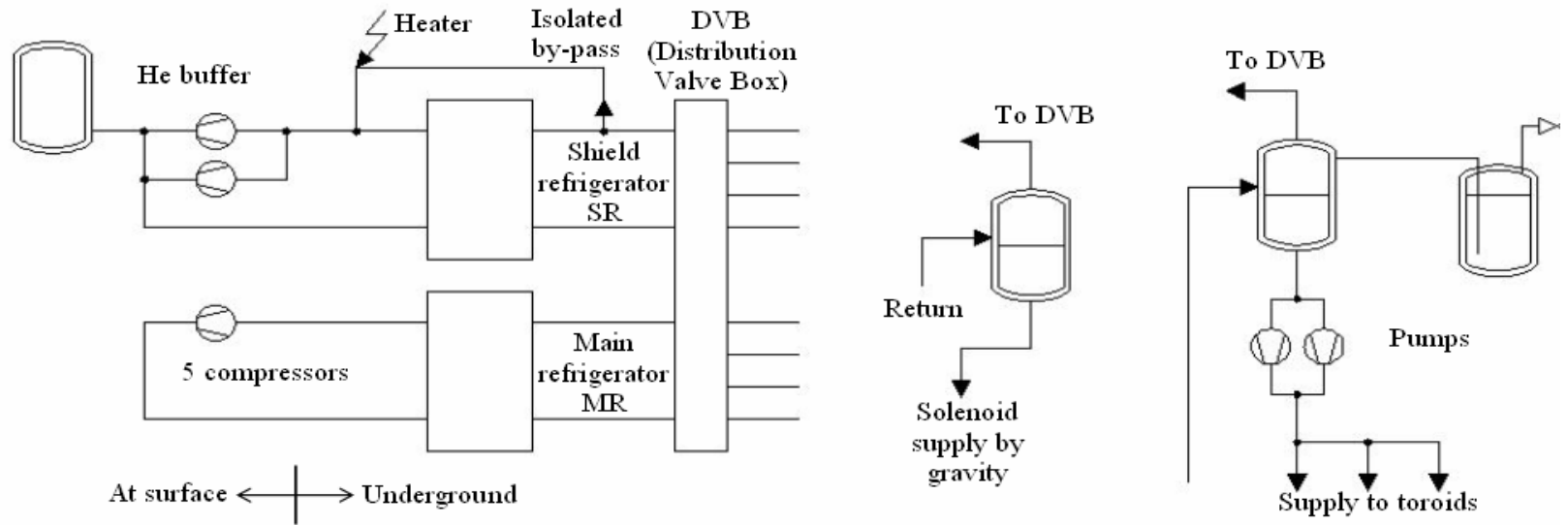

Figure 1 Emergency operation mode (MR refrigerator failure). Supply of toroids (pump) and solenoid (thermo-syphon) with stored helium. He recovery with SR shield compressor.

\section{Cryogenics for Calorimeters}

The three liquid argon calorimeter cryostats operating at $89 \mathrm{~K}$ dispose each of an expansion vessel at a higher level connected with transfer lines (flexible for the two end caps EC which can be moved and rigid ones for the central barrel) to provide sub-cooling of the liquid by hydrostatic pressure (7). Cooling of the calorimeters is done with a $\mathrm{LN}_{2}$ refrigerator with surface dewars back-up in case of failure. The $\mathrm{LN}_{2}$ is circulated in the calorimeter heat exchangers by centrifugal pumps with bypass circuits for allowing cooling directly from surface dewars. These measures have been adopted to keep the calorimeter cryostats cold at any time. In the unlikely event of their total failure or degradation of vacuum the argon pressure in the cryostat will rise and relief valves on the respective expansion vessel activate to blow out to the 500 $\mathrm{mm}$ diameter vent line connecting to the surface area. Also the nitrogen pressure relief valves of the 
respective equipment are connected to this line. In addition, in case of emergency, the liquid argon can be drained into the two adjacent $50 \mathrm{~m}^{3}$ dewars by gravity. To accelerate the emptying process centrifugal pumps can be used.

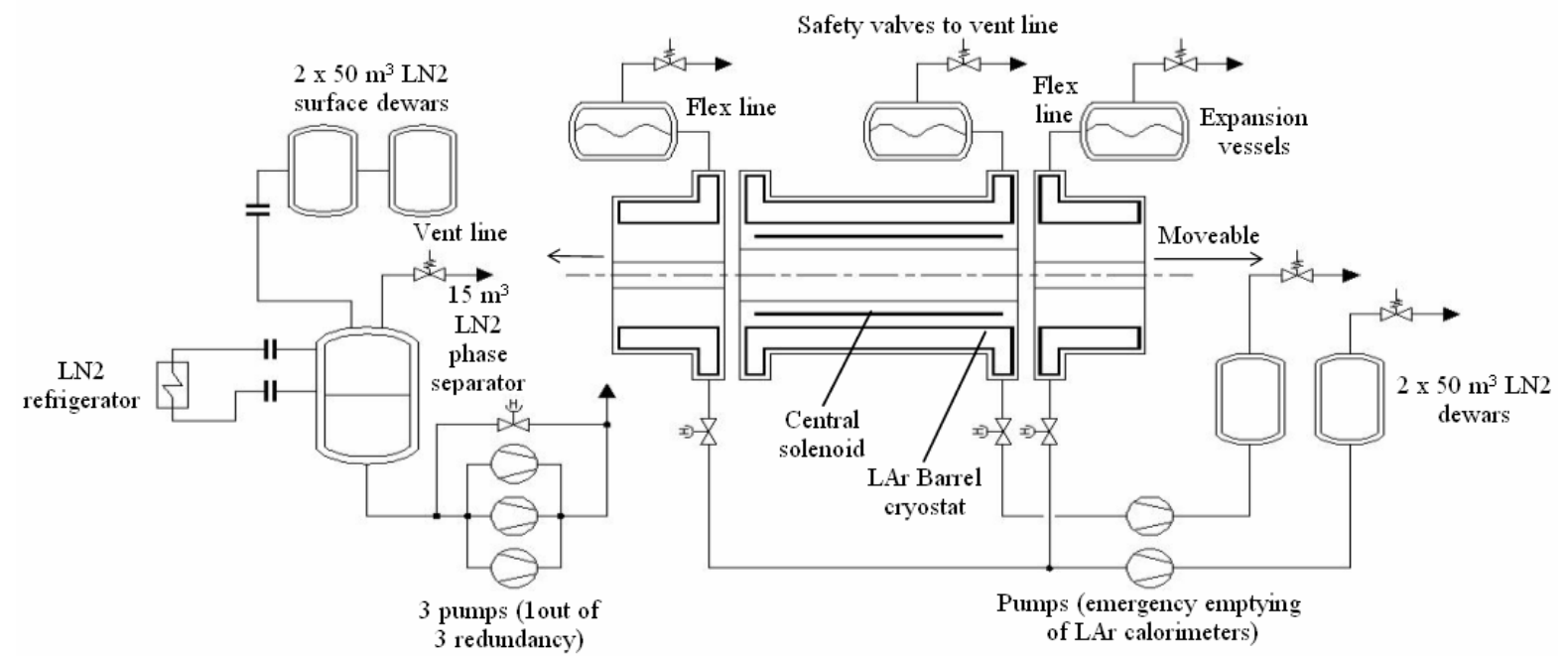

Figure 2 Redundancy in $\mathrm{LN}_{2}$ coolant supply (left) to the liquid argon cryostats (right) showing equipment for emergency drain and safety valves connecting to vent line

Services Redundancy

For vital equipment, services supply of electricity, cooling water, compressed air is equally redundant. CERN benefits from the rapid switch-over capability between the electricity networks of France and Switzerland in case of failure of either. For all of ATLAS, un-interruptable power supplies (UPS, 2 x 600 $\mathrm{kVA})$ and Diesel generators $(2 \times 900 \mathrm{kVA})$ are installed. Cryogenics uses this supply for the PLC's and the proximity cryogenics with the centrifugal coolant pumps. Two redundant air compressors with buffer volume and back-up 200 bar nitrogen batteries are installed. Two-out-of-three redundancy is applied for the water supply with 3 cooling towers and 3 water pumps. The water pumps, the air compressors and the two helium shield (SR) compressors benefit from the electricity grid switching capability.

\section{IN CASE OF ACCIDENTAL CRYOGEN LOSS}

\section{$\underline{\text { Retention areas and volumes }}$}

The entire ATLAS detector is placed on a retention area formed by trenches in the concrete floor. In case of argon spillage of the calorimeter vessels the fluid is conducted to a low point pit. Also the two $50 \mathrm{~m}^{3}$ argon and the $15 \mathrm{~m}^{3}$ nitrogen dewars are placed in pits. Ventilation ducts are installed for extraction. In case of helium loss the air-gas mixture moves upwards towards the vault of the cavern. Estimates have shown that in worst case assumptions of rapid release of the entire $11 \mathrm{~m}^{3}$ of helium the air-gas mixture expands downwards from the ceiling when warming up. The cloud with air moisture and little oxygen contents could reach the detector upper level endangering personnel working there. In order to provide a passive safety feature in parallel to the ventilation extraction systems for such severe accident the vertical access shaft PX 16 (fig. 3) is kept open at its bottom and closed at the top. The helium can escape to this $7000 \mathrm{~m}^{3}$ retention volume sufficiently large to accommodate the amount of helium stored in the cavern.

\section{Detection and Alarms}

To ensure safety of personnel by detecting abnormal situations two independent systems are installed: the "SNIFFER" and the oxygen deficiency hazard meters "ODH". The SNIFFER is multipurpose to simultaneously detect smoke from fire, flammable gas and oxygen deficiency. A network with 200 air sampling tubes are installed within the detector envelope for all gases with aspiration points for the argon calorimeters in particular at the most vulnerable feed-throughs regions. In case of detection of leaks the respective location is known. This information can be used for warning of personnel working at proximity and for the rescue and localising by the fire brigade. The ODH meters system is independent and will be installed outside the detector limits, e.g. at the floor level in retention pits and shafts and close to big vessels to detect ODH from leaks or spills. At oxygen levels of $18.5 \%$ both ODH and SNIFFER systems 
generate a level-3 alarm. A level-3 is the highest alarm level and its significance is a hazardous, serious abnormal situation or accident where people's lives may be in danger and an immediate action by the fire and rescue group is eminent.

\section{Ventilation system}

The level-3 alarm is sent with additional information to the safety control room of the rescue and fire group. An activation of the emergency operation mode of the ventilation system may be done. The ventilation system for ATLAS is rather complex and it can be categorised to provide for the following functions: Air Pressurisation, Air Ventilation and Extraction. Pressurisation is done in the underground areas and the access shaft at three different pressure levels with a difference of approximately $50 \mathrm{~Pa}$. The

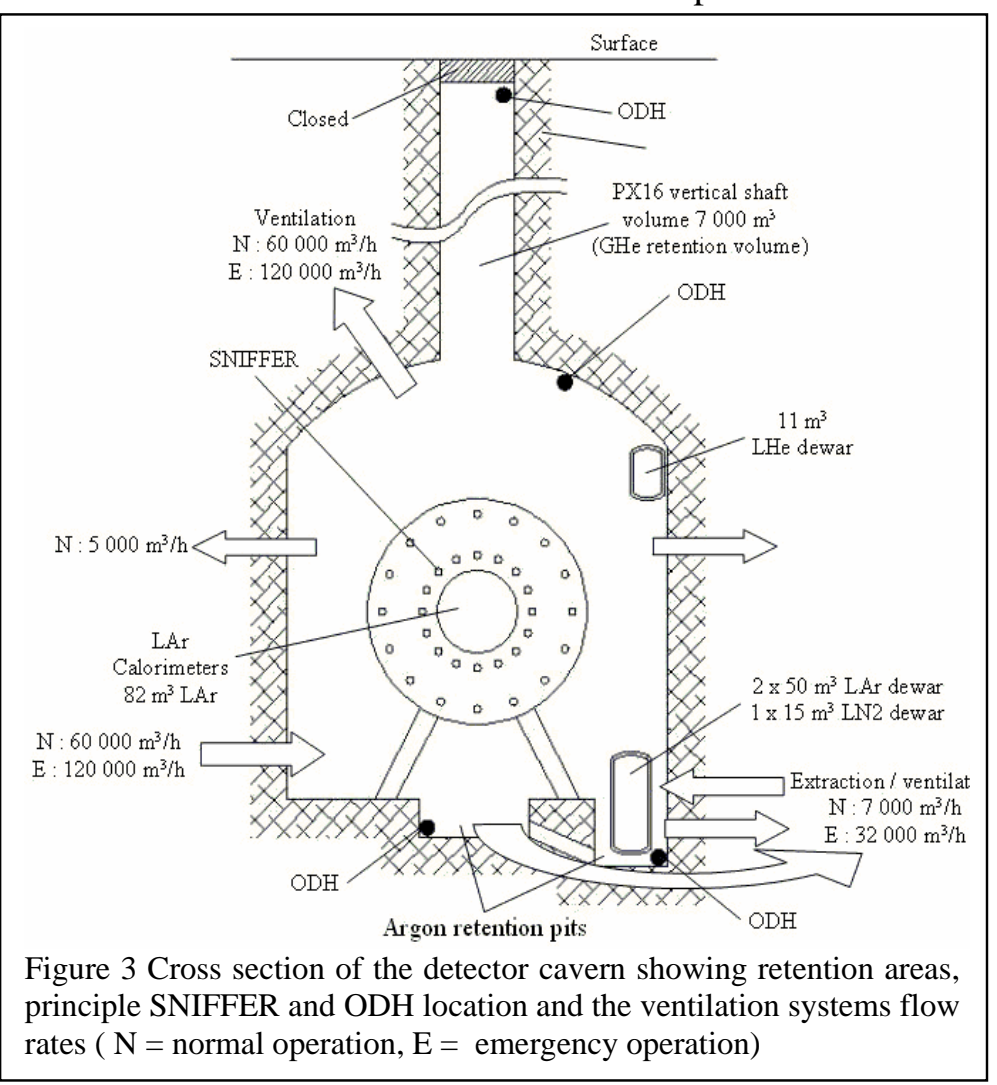
main detector cavern exhibits the lowest, slightly sub-atmospheric pressure. A permanent air extraction of $5000 \mathrm{~m}^{3} / \mathrm{h}$ with several intakes at either side of the cavern wall is made for small detector or nitrogen leaks. For the retention pits and trenches of the argon calorimeters and tanks the permanent extraction flow is $7000 \mathrm{~m}^{3} / \mathrm{h}$. In case of emergency with ODH following a leak or spill the extraction rate is increased to 32000 $\mathrm{m}^{3} / \mathrm{h}$. A large ventilation system with laminar injectors at the floor level of the cavern and two extraction vaults at the ceiling provide a permanent flow of $60,000 \mathrm{~m}^{3} / \mathrm{h}$ of air which under normal operation is partially re-circulated flow. In case of emergency (fire, helium leaks...) the flow is increased by a factor of two to $120,000 \mathrm{~m}^{3} / \mathrm{h}$ in open circuit with a $100 \%$ fresh air intake from the injectors.

\section{ACKNOWLEDGEMENTS}

Particular thanks are due to G.-P. Benincasa who is the ATLAS safety group leader for his engagement to bring the experiment to a high safety standard comprising the cryogenics part. Acknowledged are all the many contributions in matters of safety from colleagues at CERN and collaborating institutes.

\section{REFERENCES}

1 Technical Inspection and Safety Service, "The Use of Cryogenic Fluids", Safety Instructions, TIS IS 47, CERN internal report, published 1998, revision 2004.

2 Edeskuty, F.J., Stewart, W.F., "Safety in Handling of Cryogenic Fluids", Plenum Press, New York, 1996

3 Chorowski, M., Konopka, G., Riddone, G., Rybkowski, D., "Experimental Simulation of Helium Discharge into the LHC Tunnel", Proceedings ICEC 19, Grenoble, France, 2002

4 Vadon, M., Perea Solano, B., Balda, F., "Simulations of Liquid Argon Accidents in the ATLAS Cavern", Proceedings CEC, Madison, 2001

5 Delruelle, N., Haug, F., Passardi, G., ten Kate, H., "The helium cryogenic system for the ATLAS experiment", IEEE Trans. on Applied Superconductivity, 2000

6 Haug, F., Bottura, L., Broggi, F., Junker, S., "Quench induced Pressure Rise in the Cooling Pipes of the ATLAS Barrel Toroid Model", Proceedings CEC, Anchorage, 2003, to be published

7 Bremer, J., "The Cryogenic System for the ATLAS Liquid Argon Detector", Proceedings ICEC 18, Bombay, 2000 